desired species into a well defined state. The second photo-excitation step may be a Rydberg state that is field-ionized by a static electrical field, the resulting ion being captured and extracted. One other very interesting possibility for the second step, according to Karlov, would be an autoionizing state.

Laser chemistry and laser isotope sepaation have given a new stimulus to dissociation spectroscopy of molecules. C.B. Collins has demonstrated a remarkable method based on two-photon absorption that occurs through a resonant intermediate state, unstable against dissociation on the time scale of the two-photon process. The resulting products of the dissociation are photo-excited to Rydberg states that can be readily ionized for detection. In the case of caesium dimers two relatively broad absorption bands were found, corresponding to the population of the two fine structure components of the $5^{2} \mathrm{D}$ states. The respective influence of predissociation and dissociation was demonstrated.

It is well known that the formation and loss rates of cluster ions play an important role in aeronomy. D. Smith presented a survey of very recent results, performed by a temperature variable selected ion flow tube, on the measurements of rate coefficients for many association reactions of several ion species such as $\mathrm{NO}^{+}$or $\mathrm{CH}_{3}{ }^{+}$. Relevance to interstellar molecular synthesis was also discussed. Two-body and three-body ion-ion neutralisation has been carefully and systematically observed, and the relevance of these data on atmosphere de-ionization was discussed. This field is developing at an impressive rate.

$J$. Uhlenbusch described his new results on production of plasmas by $\mathrm{CW}$ lasers. Such discharges, called continuous optical discharges (COD), are produced without any electrodes and are characterized by a low level of impurities. They seem to be very well suited for plasma chemical applications as well as welding, cutting and tempering. The COD plasma is obtained by focusing the beam of a powerful $\mathrm{CO}_{2}$ laser into a high pressure chamber (up to several hundred bars). By using very refined optical diagnostics, the main characteristics of the plasma have been carefully measured over an electron density range from $10^{17}$ $-10^{18} \mathrm{~cm}^{-3} . \mathrm{T}_{\mathrm{e}}$ varies over $13000-22000 \mathrm{~K}$.

F. Howorka presented the situation of our knowledge of transport of ions in gases under the influence of electrical fields. The Innsbruck group has actively contributed to this area. Among many interesting developments, Howorka demonstrated how exact mobility measurements allow the determination of the interaction potential of ions drifting in a given gas.

Europhysics Conference Abstracts, Vol. $4 \mathrm{D}$.

\section{Manus, Gif-sur-Yvette}

\title{
Laser Doppler Anemometry
}

\section{E.O. Schulz-DuBois, Kiel and Göttingen}

(Respectively, Institute for Applied Physics and Max-Planck Institute for Research on Fluid Dynamics)

In the past few years, laser applications of increasing complexity and sophistication have begun to make their way from research to development laboratories and from there into engineering practice, industrial production and marketing. Laser Doppler anemometry is a case in point. In what follows, basic principles, the state of the art and some typical applications will be reviewed. Apologies are made to laser Doppler specialists for what may seem to be a disregard for many of their ingenious schemes and inventions, but interested readers may go more deeply into the subject by consulting references 1,2 and 3 .

\section{The Beginning: Optical Heterodyning}

During the development of the first gas lasers, beats between adjacent longitudinal modes were observed. For an $L=1 \mathrm{~m}$ long laser, for example, the beat frequency is $c / 2 L=150 \mathrm{MHz}$, and it is due to laser modes which differ by 1 in the number of half wavelengths in the standing wave pattern between mirrors. This suggested that with laser beams of adequate spectral purity and wavefronts sufficiently parallel to one another, differences between optical frequencies could be obtained much like intermediate frequencies are obtained by heterodyning radio frequencies. Further exploration of this possibility led to the evolution of heterodyne spectroscopy. Laser light impinges on a sample from which Stokes or anti-Stokes shifted light is scattered through Rayleigh, Brillouin, or Raman effect. Superposition of scattered light with part of the original laser light on a photodetector results in an electrical signal at the difference frequency.

H. Z. Cummins, one of the originators of heterodyne spectroscopy, also initiated laser Doppler anemometry. In 1964, he and his coworkers directed a laser beam at particles that were being carried along by a laminar fluid flow. Owing to the particle velocity, the scattered light frequency was Doppler shifted. Superposing the scattered light with part of the original laser beam on a photodetector, they observed Doppler frequency shifts in the $\mathrm{kHz}$ range. They moved the spot from which scattered light was collected relative to the flow geometry and, in this way, were able to trace out the parabolic Poiseuille velocity profile, characteristic of laminar flow in round tubing.

The heterodyne method used in laser velocimetry, in the jargon of the field, is called the reference beam method. In association with modern optical and electronic equipment, it is popular for a number of applications, but it suffers the drawback of low sensitivity. For heterodyne detection, scattered light and the original beam should have coplanar wavefronts over the area of the photodetector. That can be accomplished only for a small detector area or, equivalently, for small acceptance aperture.

Other methods of laser velocimetry do not depend on optical heterodyning directly. Their modus operandi may be discussed on the basis of seemingly different, yet fundamentally equivalent principles.

Basic Principle: Time-of-Flight Measurement

In any kind of velocimetry, a distance is marked by elements of the apparatus and the time that the object under study takes to travel that distance is measured. Dual focus velocimetry utilizes two focal regions of laser beams as sketched in Fig. 1. Typical dimensions are $10 \mu \mathrm{m}$ for the beam diameters at their waist and $300 \mu \mathrm{m}$ for their separation. These beams represent light gates. If a particle (city air or clean water usually contain enough particles for the purpose, otherwise artificial "seeding" may be called for) passes both focal regions in succession it will cause a burst of scattererd light in each. Photodetection is arranged to receive light signals from both focal regions in separate channels and the time of passage is equal to the time delay between pulses. The time difference may be obtained for example, by a time-tovoltage converter (originally developed for nuclear physics instrumentation) or by cross correlation. The fast digital electronic correlator used for this purpose will be discussed below.

\section{Fig. 1. - Principle of laser dual focus} velocimeter.

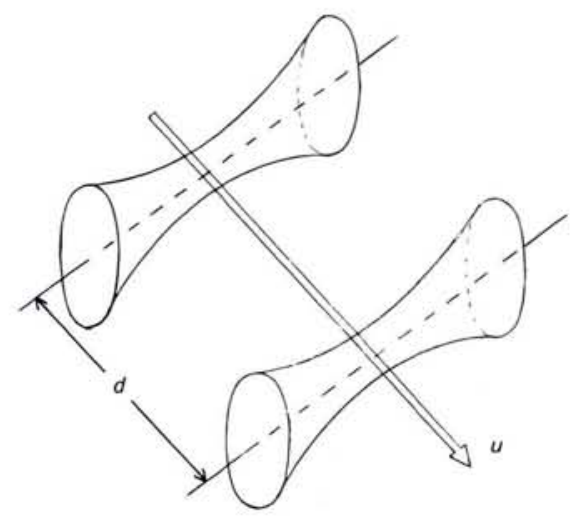


From the dual focus geometry it is obvious that this velocimeter obtains signals only when the velocity vector lies in the plane defined by both beams. Thus it has a high angular resolution, but is not too well suited to highly turbulent flows. Nevertheless a more detailed appraisal of dual focus velocimetry shows that it is largely insensitive to flare, i.e. the reflection of laser light at nearby sufaces, and hence may be used for studying the flows inside rotating machinery like jet turbines.

\section{Multiplied Time-of-Flight: Cross-Beam}

\section{Arrangement}

Instead of two separate light gates one may also use a periodic array of light gates. A periodic arrangement of light intensity (mean square of the electric field) is easily realized by two light beams of the same frequency crossing at an angle $\alpha$. The resulting interference fringes have a lattice separation constant $d$ given by the Bragg formula:

$$
d=\lambda /(2 \sin \alpha / 2)
$$

and are oriented perpendicular to the plane of the beams. If $\overline{k_{1}}$ and $\overline{k_{2}}$ are the wave vectors of the beams, the interference fringe planes are given by

$$
\left(\overline{k_{1}}-\overline{k_{2}}\right) \cdot\left(\bar{r}-\overline{r_{0}}\right)=2 n \pi
$$

where $n$ in an integer and $r_{0}$ the coordinate origin. Taking the finite cross section of the beams into account, the fringe pattern tapers off as one moves away from the centre point, resulting in a sampling volume of ellipsoidal shape as shown in Fig. 2.

A small particle passing the fringes on trajectories A or B, will scatter light of intensity that is proportional to the incident intensity, and with a time function as shown in Figs. $3 \mathrm{~A}$ and $\mathrm{B}$, respectively. After photodetection and highpass filtering, the electronic signal in either case wil look like Fig. $3 \mathrm{C}$. The time, $\tau$, from one voltage maximum to the next is, obviously, the time the particle takes to move from one fringe to the next. Hence:

$$
\tau=d / u
$$

where $u$ is the particle velocity. More precisely, $u$ is the velocity component perpendicular to the fringe planes. In other words, the crossbeam LDA measures a Cartesian velocity component. Formally, this is shown by differentiating equation (2), yielding the Doppler (radian) frequency difference:

$$
\omega_{D}=\left(\overline{k_{1}}-\overline{k_{2}}\right) \cdot \bar{u} .
$$

Note that so far the signal has been explained in terms of the light scattered from a stationary interference pattern, without explicit reference to Doppler frequency shift.

\section{Equivalent Viewpoint: Optical Doppler Effect}

It is possible, however, to interpret the signal as a genuine Doppler effect. The incident laser fields may be considered as proportional to:

$$
\exp \left[i\left(\omega_{0} t-\overline{k_{1}} \cdot \bar{r}\right)\right], I=1,2
$$

A particle path is:

$$
\bar{r}=\bar{r}_{\mathrm{o}}+\overline{u t}
$$

and by inserting (6) into (5) one finds two Doppler shifted laser frequencies

$\omega_{1}=\omega_{0}-\overline{k_{1}} \cdot \bar{u}$ and $\omega_{2}=\omega_{0}-\overline{k_{2}} \cdot \bar{u}$ (7) respectively, which the particle receives and reradiates. Note, however, that the radiating particles moves while the detector is stationary. This is equivalent to a stationary radiating particle and the detector moving with velocity $-\bar{u}$. Hence the detector receives two frequencies which are shifted once more,

$$
\begin{aligned}
& \omega_{1}^{\prime}=\omega_{0}-\left(\overline{k_{1}}-\overline{k_{D}}\right) \cdot \bar{u} \text { and } \\
& \omega_{2}^{\prime}=\omega_{0}-\left(\overline{k_{2}}-\overline{k_{D}}\right) \cdot \bar{u}
\end{aligned}
$$

$\left(\overline{k_{D}}\right.$ is the wave vector in the direction from particle to detector). The difference between $\omega_{1}^{\prime}$ and $\omega_{2}^{\prime}$ is the Doppler frequency difference $\omega_{D}$ previously given in equation (4). Remarkably, $\omega_{D}$ does not depend on $\bar{k}_{D}$, i.e. the direction of light reaching the detector. This implies that reception with wide aperture is possible without detrimental effects on signal quality. In fact, it is this feature which results in high sensitivity of crossbeam LDA systems.

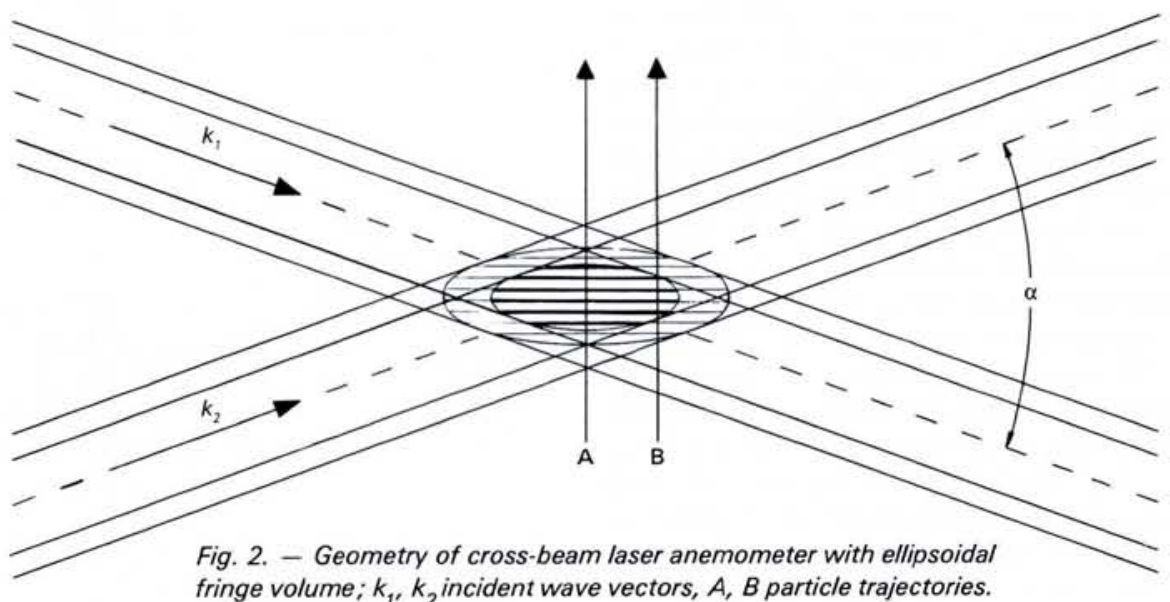

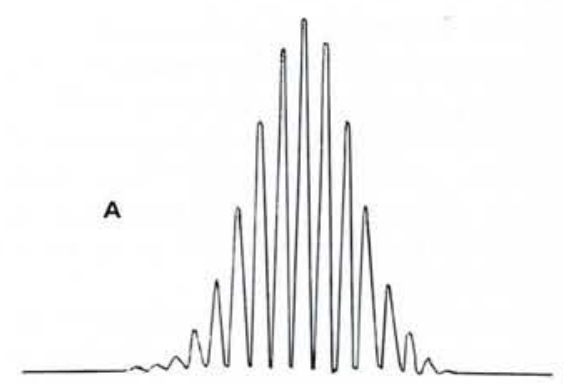
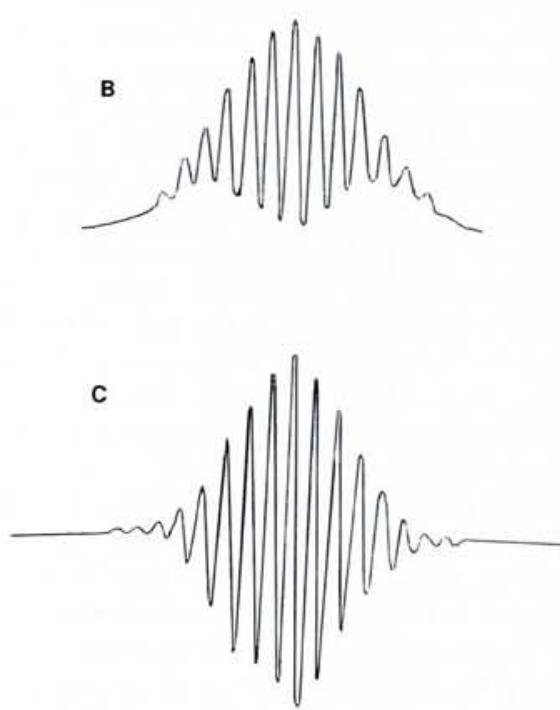

Fig. 3. - Scattered light intensity versus time; A central passage, $B$ off-centre passage of parti cle, C high-pass filtered electronic signal, the socalled Doppler burst.

\section{Counter and Tracker}

The object of electronic processing is to extract frequency information from signals like Fig. 3C. The choice of equipment depends on several factors. For example, assume there are very few particles in the flow field so that the photodetector receives well separated Doppler "bursts". Also assume a good signal to noise ratio in the received signal. Then a counter-type processor is preferred, recording only signals exceeding a given threshold. The time between zero crossings is clocked and, as a further precaution, the time for (say) 8 Doppler cycles is compared with that for 5 , and the measurement is accepted only if the time ratio is $1.6 \pm$ (say) 0.1 . The result is available at the output in analog and digital form and it is updated whenever a new valid measurement is made.

If there are many particles in the stream, the Doppler bursts tend to overlap and a quasicontinuous signal results. It shows amplitude fluctuations caused by the particle statistics and some phase jitter, since successive particles are not at the fringe maxima at the same time. In this case a tracker is used to advantage. It consists of a voltage controlled oscillator which produces an artifical Doppler signal of cons- 
tant amplitude. A feedback circuit (a phase locked loop) adjusts frequency and phase of this oscillator to track that of the received photodetector signal. This involves some inertia to overcome intervals of inadequate received signal, but a continuous output is available in terms of the Doppler frequency and analog voltage.

\section{Very Few Photons: the Digital Cor-} relator

The Digital Correlator works well even with very low received light levels, fairly accurate velocity estimates being obtained from as little as 50 detected photons. A further advantage is its "democratic" operating principle, i.e. all received photons are treated alike. Hence statistical and information analysis may be applied to obtain the limiting accuracy of results. By comparison, threshold devices which are used in the counter and tracker introduce a degree of arbitrariness, e. g. in the level in threshold devices, which makes a similar assessment of results difficult.

Photomultiplier pulses, each one representing a single detected photon, are converted to pulses of standard amplitude and duration, and the number registered in equal time slots is recorded. Using a shift register delay line, many multipliers and stores, the digital, discrete, truncated form of the intensity autocorrelation function is accumulated in the stores. If $/(t)$ is light intensity, the function so obtained is, except for unessential factors, an estimate of the autocorrelation function:

$$
G(\tau)=\lim _{T \rightarrow \infty} \frac{1}{T} \int_{0}^{T} /(t) /(t+\tau) d t .
$$

By the Wiener-Khintchine theorem, the Fourier transformed of $G(\tau)$ is the spectrum. In the present case, the spectrum contains a maximum at the most prominent Doppler frequency $\omega_{D}$. In addition, there is instrumental broadening due to the finite width of laser beams and possibly further broadening due to velocity fluctuations, i.e. turbulence.

A trade-off is possible between measurement time and accuracy. For best accuracy, one uses a long accumulation time, perhaps many seconds, to overcome the effects of digitalization and genuine noise. On the other hand, fair estimates of velocity may be obtained after short accumulation, e. g. over a fraction of a microsecond. A number of algorithms exist to extract velocity information out of correlograms in various circumstances. This is not restricted to average velocity but includes higher moments of the velocity distribution as well.

\section{State of the Art}

Laser Doppler anemometry offers a nonintrusive method of measuring fluid velocities, suitable for hostile environments where material probes cannot endure, e. $\mathrm{g}$. in flames, explosions, combustion, acids. Besides the optical systems discussed reference beam, dual focus, cross-beam there are several others, including telescopic with up to $1 \mathrm{~km}$ range and glass fibre systems capable of measuring inside blood vessels. Other refinements in optical systems allow the flow direction to be distinguished (referring to equation (4), positive and negative $\omega_{D}$ cannot be distinguished experimentally) and the simultaneous measurement of two or three components. Velocities measured range from $0.1 \mu \mathrm{m} / \mathrm{s}$ to $1000 \mathrm{~m} / \mathrm{s}$. Once assembled, equipment works reliably and can be operated by unskilled labour.

On the other hand, further development may still be required if one wants to measure at very large/very small dimensions, if extremely high/extremely low velocities are expected, if high accuracy (relative error smaller than $10^{-3}$ ) or good data on the statistics of turbulence are asked for, or if flow at not easily accessible locations is to be studied. Examples are measurements in the boundary layer at the wing of airborne aircraft, near the propeller of a ship while moving on the ocean, or between blades of high-speed turbines.

Not surprisingly, laser Doppler anemometry has revitalized several areas of fluid mechanics research. Besides the topics mentioned already, it provides a means of studying two-phase flows, for example bubbles in water, where the velocities of both components may be determined separately, flames (with graphite particles), sediment transport in rivers and oceans, powdered materials in air and others. Another field is the study of turbulence which is now possible with a higher degree of sophistication. An area that has attracted the curiosity of many physicists is that of hydrodynamic instabilities ${ }^{4}$ ). In some well-defined geometries, the transition from laminar to turbulent flow occurs via several intermediate modes of fluid flow, characterized by well-defined frequencies and wave numbers. A first step towards interpreting these phenomena is a theoretical model due to Landau which is formally analogous to the mean-field theory of continuous phase transition due to Landau and Lifschitz.

\section{BIBLIOGRAPHY}

1. Durst F., Melling A. and Whitelaw J.H., Principles and Practice of Laser Doppler Anemometry (Academic Press) 1976.

2. Durrani T.S. and Greated C.A., Laser Systems in Flow Measurement (Plenum Press) 1977.

3. Cummins H.Z. and Pike E.R., (Eds.) Photon Correlation Spectroscopy and Velocimetry (Plenum Press) 1977.

4. Swinney H.L. and Gollub J.P. "The Transition to Turbulence", Physics Today 31 (1978) 8, p. 41.

\section{A Breadth of Vision}

Wolfgang Gentner, founder member of EPS and member of the first Executive Committee, has died at the age of 74. A keen promoter of European co-operation in physics, Gentner's influence has been felt in the development of a number of European scientific collaborations.

Basically a nuclear physicist, he was one of the first in the Federal Republic of Germany to look towards higher energies, starting the first cyclotron there of $20 \mathrm{MeV}$ at Heidelberg. He was a vigorous supporter of CERN and for five years headed the Division that brought the CERN $600 \mathrm{MeV}$ sychro-cyclotron into operation and launched the programme of physics research. He served on the CERN Scientific Policy Committee and as delegate to Council, being elected President from 1972-74. His particular interest was in highly unstable nuclei and excited states.

$\mathrm{He}$ was also interested in geology and in the isotopic concentrations of matter and the clues they give to the origin of the Universe. With his collaborators, he was the first in Europe to study moon rocks. He became an expert on dating and was active in introducing modern physical techniques
- tracer methods, thermally induced photoluminescence, track sensitive methods, etc., to the solution of archaeological puzzles. He was able to trace the origins of the silver to be found in old coins and by applying tracer techniques to identifying the origins of pottery, proved that trade in ancient times was much freer than had been supposed.

With his breadth of interests, he was an energetic promoter of the inter-disciplinary and cultural aims of the European Science Foundation. He was closely involved in the foundation of the Laue-Langevin Institute in Grenoble and equally enthusiastic over the creation of Emblab. He had special connexions with Israel and was his country's representative on the Minerva collaboration with Rehovot.

Founder of the Max-Planck-Institut für Kernphysik in 1958, he was until 1972 its Executive Director. In all his activities, he was particularly well-known for his encouragement of young physicists, open to the unconventional approach and welcoming personalities of widely different background. His breadth of vision will remain an inspiration. 